\title{
A Geodesic Voting Shape Prior to Constrain the Level Set Evolution for the Segmentation of Tubular Trees ${ }^{\star}$
}

\author{
Youssef Rouchdy and Laurent D. Cohen \\ CEREMADE, UMR 7534, Université Paris Dauphine, \\ 75775 Paris Cedex 16, France \\ youssef.rouchdy@gmail.com, cohen@ceremade.dauphine.fr
}

\begin{abstract}
This paper presents a geodesic voting method to segment tree structures, such as retinal or cardiac blood vessels. Many authors have used minimal cost paths, or similarly geodesics relative to a weight potential $\mathrm{P}$, to find a vessel between two end points. Our goal focuses on the use of a set of such geodesic paths for finding a tubular tree structures, using minimal interaction. This work adapts the geodesic voting method that we have introduced for the segmentation of thin tree structures to the segmentation of tubular trees. The original approach of geodesic voting consists in computing geodesics from a set of end points scattered in the image to a given source point. The target structure corresponds to image points with a high geodesic density. Since the potential takes low values on the tree structure, geodesics will locate preferably on this structure and thus the geodesic density should be high. Geodesic voting method gives a good approximation of the localization of the tree branches, but it does not allow to extract the tubular aspect of the tree. Here, we use the geodesic voting method to build a shape prior to constrain the level set evolution in order to segment the boundary of the tubular structure. We show results of the segmentation with this approach on 2D angiogram images and 3D simulated data.
\end{abstract}

\section{Introduction}

In this paper we present a novel method for the segmentation of tree structures. These methods are based on minimal paths with a metric designed from the images and can be applied to the segmentation of numerous structures, such as: microglia extensions; neurovascular structures; blood vessel; pulmonary tree. The vascular tree is modeled as a tubular structure. We consider among the methods used to segment the vascular tree three classes of approaches according to the method used to extract the tubular aspect of the tree: surface models; centerline based models; and 4D curve models. The first category extracts directly the surface of the vessel, see [1. For the second approach, centerlines based models, centerlines are extracted first and a second process is required to segment the

\footnotetext{
* This work was partially supported by ANR grant MESANGE ANR-08-BLAN-0198.
} 
vessel surface, see [2]. The last approach, 4D curve model, consists in segmenting the vessel centerlines and surfaces simultaneously as a path in a (3D+radius) space [344. For a review of these methods, see [5]6.

Minimal paths techniques were extensively used for extraction of tubular tree structures. These approaches are more robust than the region growing methods, particularly in the presence of local perturbations due to the presence of stenosed branches of the tree or imaging artefacts where the image information might be insufficient to guide the growing process. Several minimal path techniques have been proposed to deal with this problem 7819. These techniques consist in designing a metric from the image in such a way that the tubular structures correspond to geodesic paths according to this metric. Solving the problem from the practical point of view consists of a front propagation from a source point within a vessel which is faster on the branches of the vascular tree. These methods required the definition by the user of a starting point (propagation source) and end points. Each end point allows to extract a branch of the tree as a minimal path from this point to the source point, the points located on the minimal path are very likely located on the vessel of interest. Few works have been devoted to reduce the interaction of the user in the segmentation of tree structure to the initialization of the propagation from a single point. Authors of [10] defined a stopping criteria from a medialness measure, the propagation is stopped when the medialness drops below a given threshold. This method might suffer from the same problem as the region growing, the medialness might drop below the given threshold in the presence of pathology or imaging artefacts. Wink et al. 11] proposed to stop the propagation when the geodesic distance reaches a certain value. However, this method is limited to the segmentation of a single vessel and the definition of the threshold of the geodesic distance is not straightforward. Cohen and Deschamps 12 proposed to stop the propagation following a criterion based on some geometric properties of the region covered by the front. In 9 , assuming the the total length of the tree structure to be visited is given, the stopping criteriuon is based on the Euclidean length of the minimal path.

Li et al. 4] proposed a 4D curve model with a key point searching scheme to extract multi-branch tubular structures. The vascular tree is a set of $4 \mathrm{D}$ minimal paths, giving 3D centerlines and width. While this method has the advantage to segment vessel centerlines and surfaces simultaneously, it requires the definition of eight parameters. One point inside the tubular structure and the radius are used to initialize the Fast marching propagation, three parameters are used to set the Fast Marching potential and three distance parameters limit the propagation to the inside of the tubular structure to avoid leakage outside the tree. These last three parameters may require an important intervention of the user since they are crucial to extract the whole structure. If these distance parameters are not suitable, parts of the tree structure may be missed during the propagation.

In this paper, we present a method to extract tree structures without using any a priori information. Furthermore, the user has to provide only a single point on the tree structure. The method is generic: it can be used to extract any type of tree structure in $2 \mathrm{D}$ as well as in $3 \mathrm{D}$. It is based on the geodesic voting 
method introduced in [1314]. It consists in computing geodesics from a given source point to a set of end points scattered in the image. The target structure corresponds to image points with a high geodesic density. The geodesic density is defined at each pixel of the image as the number of geodesics that pass over this pixel. Since the potential takes low values on the tree structure, geodesics will locate preferably on this structure and thus the geodesic density should be high on the tree structure. While the original voting method allows to extract tree structures it does not permit to extract the walls of the vessels. Here, we introduce a shape prior constraint constructed from the geodesic voting method to constrain the evolution of a level set active contour in order to extract the walls of the tree. We use a Bayesian approach to introduce this prior into the level set formulation. We end up with a minimization problem of a global energy composed of two terms. The first term corresponds to a deformation energy for a standard region based level set method and the second term introduces the shape prior constraint. In Section 2, we present the tools needed in Section 3 to introduce the new geodesic voting method. In Section 4 , we applied our approach to the segmentation of vessels from $2 \mathrm{D}$ angiogram images and $3 \mathrm{D}$ simulated data.

\section{Background}

\subsection{Minimal Paths}

In the context of image segmentation Cohen and Kimmel proposed, in [15], a deformable model to extract contours between two points given by the user. The model is formulated as finding a geodesic for a weighted distance:

$$
\min _{y} \int_{0}^{L}(w+P(y(s))) \mathrm{d} s,
$$

where $s$ is the arclength, $L$ is the length of the curve and the minimum is considered over all curves $y(s)$ traced on the image domain $\Omega$ that link the two end points, that is, $y(0)=x_{0}$ and $y(L)=x_{1}$. The constant $w$ imposes regularity on the curve. $P>0$ is a potential cost function computed from the image, it takes lower values near the edges or the features. For instance $P(y(s))=I(y(s))$ leads to darker lines while $P(y(s))=g(\| \nabla I||)$ leads to edges, where $I$ is the image and $g$ is a decreasing positive function.

To compute the solution associated to the source $x_{0}$ of this problem, [15] proposed a Hamiltonian approach: Find the geodesic weighted distance $\mathrm{U}$ that solves the eikonal equation :

$$
\|\nabla \mathrm{U}(x)\|=w+P(x) \quad \forall x \in \Omega
$$

The ray $y$ is subsequently computed by back-propagation from the end point $x_{1}$ by solving the Ordinary Differential Equation (ODE): $y^{\prime}(s)=-\nabla \mathrm{U}(y)$. To solve the eikonal equation (2), we use the Fast Marching algorithm introduced in 16. The idea behind the Fast Marching algorithm is to propagate the wave 
in only one direction, starting with the smaller values of the action map $U$ and progressing to the larger values using the upwind property of the scheme. Therefore, the Fast Marching method permits to solve the equation (2) in complexity $O(n \log (n))$, where $n$ is the number of grid points, for details see [16[15].

\subsection{Geodesic Voting for Segmentation of Tree Structures}

We have introduced in [13]14 a new concept to segment a tree structure from only one point given by the user in the tree structure. This method consists in computing the geodesic density from a set of geodesics extracted from the image. Assume you are looking for a tree structure for which a potential cost function has been defined as above and has lower values on this tree structure. First we provide a starting point $x_{0}$ roughly at the root of the tree structure and we propagate a front in the whole image with the Fast Marching method, obtaining the minimal action U. Then assume you consider an end point anywhere in the image. Backtracking the minimal path from the end point you will reach the tree structure somewhere and stay on it till the start point is reached. So a part of the minimal path lies on some branches of the tree structure. The idea of this approach is to consider a large number of end points $\left\{x_{k}\right\}_{k=1}^{N}$ on the image domain, and analyze the set of minimal paths $y_{k}$ obtained. For this we consider a voting scheme along the minimal paths. When backtracking each path, you add 1 to each pixel you pass over. At the end of this process, pixels on the tree structure will have a high vote since many paths have to pass over it. On the contrary, pixels in the background will generally have a low vote since very few paths will pass over them. The result of this voting scheme is what we can call the geodesic density. This means at each pixel the density of geodesics that pass over this pixel. The tree structure corresponds to the points with high geodesic density. The set of end points for which you consider the geodesics can be defined through different choices. This could be all pixels over the image domain, random points, scattered points according to some criterion, or simply the set of points on the boundary of the image domain, see [14]. We define the voting score or the geodesic density at each pixel $p$ of the image by

$$
\mu(p)=\sum_{k=1}^{N} \delta_{p}\left(y_{k}\right)
$$

where the function $\delta_{p}(y)$ returns 1 if the path $y$ crosses the pixel $p$, else 0 . Once the geodesic voting is made, the tree structure is obtained by a simple thresholding of the geodesic density $\mu$. As shown in Figure 1, the contrast between the background and the tree is large and the threshold can be chosen easily. We used for all experiments the following value

$$
T h=\frac{\max (\text { geodesic density })}{100}
$$



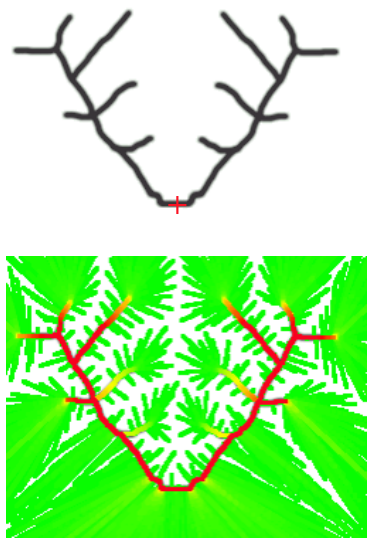
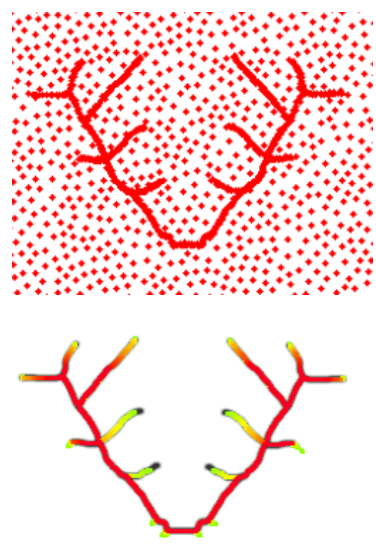
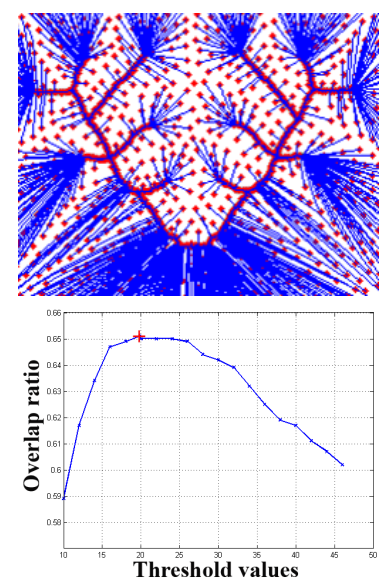

Fig. 1. Geodesic voting. First row: the left panel shows the synthetic tree, the red cross represents the root of the tree; the center panel shows the set of end points (here farthest points, see [14]); the right panel shows in blue the geodesics extracted from the set farthest points to the root. Second row: the left panel shows the geodesic density; the center panel shows the geodesic density after thresholding; the right panel plots the effect of the variation of the threshold on the overlap ratio, the red cross represents the value $T h$ (given by the equation (4) ).

as threshold to extract the tree structure using the voting maps. Figure1 (panel: second row on the right) shows the effect of the threshold on the overlap ratid 1 that measures the similarity between the the manually segmented data $A$ and the segmentation result $B$. This figure shows that the threshold can be chosen in a large range that contains the threshold $T h$, given by the equation (4).

\subsection{Active Contours without Edges}

In this section we describe the level set method that we will use in the next section to introduce our active contour model. The active contour models consist in evolving a curve (2D case) or surface (3D) constrained by image-based energy toward the target structure. Chan and Vese [17. proposed a region based model adapted to segment an image with poor boundaries (edge information). This model is a piece-wise constant approximation of the Mumford and Shah functional [18:

$$
\begin{aligned}
& \mathcal{V}\left(\phi, c_{1}, c_{2}\right)=\int_{\Omega}\left(\lambda_{1}\left(u_{0}-c_{1}\right)^{2} H_{\epsilon}(\phi)+\lambda_{2}\left(u_{0}-c_{2}\right)^{2}\left(1-H_{\epsilon}(\phi)\right)+\right. \\
& \left.\mu \delta_{\epsilon}(\phi)|\nabla \phi|+\nu H_{\epsilon}(\phi)\right) \mathrm{d} x,
\end{aligned}
$$

\footnotetext{
${ }^{1}$ The overlap ratio is defined by the relation: $\mathrm{O}(A, B)=\frac{2|A \cap B|}{|A|+|B|}$, where $|A|$ and $|B|$ are respectively the number of the foreground voxels in the image $A$ and $B .|A \cap B|$ is the number of voxels in the shared regions (intersection of the foreground of the two images)
} 
where $\phi$ defines the boundary as its zero level set; $\Omega$ is the image domain; $u_{0}$ is a given image function; $\lambda_{1}, \lambda_{2}, \nu$, and $\mu$ are positive parameters; $c_{1}$ and $c_{2}$ are two scalar constants used to separate the image into two regions of constant image intensities. The two last terms in the equation introduce regularization constraints, where $H_{\epsilon}$ and $\delta_{\epsilon}$ are respectively the regularized Heaviside and Dirac functions, in this work they are approximated by:

$$
H_{\epsilon}(\tau)=\frac{1}{2}\left(1+\frac{2}{\pi} \operatorname{artang}\left(\frac{\tau}{\epsilon}\right)\right) ; \quad \delta_{\epsilon}(\tau)=\frac{1}{\pi} \frac{\epsilon}{\epsilon^{2}+\tau^{2}} .
$$

\section{From the Voting Tree to the Tubular Tree}

While the Chan and Vese energy constraint introduces regularization to smooth the level set funcion $\phi$ and to deal with noise, it does not introduce a bias towards the target structure. Bayesian models were proposed in the literature to incorporate prior knowledge about the target structure to constrain the evolution of the level set 19. The first level set method with prior knowledge about shape was introduced by Leventon et al. [19. Recent improvements of this approach were proposed for example in [20]. The geodesic voting method described in Section 2.2 gives a good approximation of the localization of each branch of the tree.

In this section we introduce a shape prior constraint using a Bayesian framework to segment the walls of the tree structure. The idea is to use the geodesic voting method to construct the shape prior that constrains the evolution of the level set propagation. After thresholding the geodesic density $\mu$ defined by the equation (3) we get an approximation of the target tree structure as explained in Section 2.2. However this geodesic density does not allow to extract the tubular aspect of the tree. Indeed the thresholded geodesic density gives only an approximation of the centerlines of the tree structure. Our aim here is to use this rough tree skeleton to build a prior that constrains the evolution of level set active contour in order to extract the boundary of the tree.

From now on we call the voting tree the tree structure obtained after thresholding the geodesic density. To construct the shape prior from the voting tree we use the largest radius of the tubular structure. The largest radius is obtained from the target image. It does not have to be precise: it is sufficient to inspect the target tree visually and to give an approximate value. A uniformly tubular tree containing the target tree structure is obtained by morphological dilation of the voting tree with a radius that corresponds to the largest radius of the tubular tree. The prior that we will use to constrain the level set method corresponds to the signed distance from the boundary $\mathcal{S}$ of the tubular tree obtained after dilatation, which we denote $\tilde{\phi}$. The signed distance $\tilde{\phi}$ is defined by:

$$
\tilde{\phi}(x)=\left\{\begin{array}{c}
D(x), \quad \text { if } \quad x \text { is inside } \mathcal{S}, \\
-D(x), \text { otherwise, }
\end{array}\right.
$$

where $D$ is a distance from $S: D(x)=\inf _{y \in \mathcal{S}} \mathrm{d}(x, \mathcal{S})$ with d a given metric, we use in this work the Euclidean metric. The distance $\tilde{\phi}$ is then used to constrain the 
level set evolution in the target image. Let $\mathbb{P}(\phi \mid \tilde{\phi}, u)$ be the posterior probability of the level set $\phi$ given the image function $u$ and the level set shape prior. The Bayesian formulation of this probability is given by Bayes' theorem:

$$
\mathbb{P}(\phi \mid \tilde{\phi}, u)=\frac{\mathbb{P}(\tilde{\phi}, u \mid \phi) \mathbb{P}(\phi)}{\mathbb{P}(\tilde{\phi}, u)} \propto \mathbb{P}(\tilde{\phi} \mid \phi) \mathbb{P}(u \mid \phi) \mathbb{P}(\phi)
$$

where $\mathbb{P}(\tilde{\phi} \mid \phi)$ is the shape prior term, we suppose that this probability follows a Gaussian distribution and that $\mathbb{P}(u \mid \phi) \mathbb{P}(\phi)$ is derived from the Chan and Vese model, see equation (5). Therefore, the maximum of the posterior probability (7) is equivalent to the lowest energy of the $(-\log )$ functional, and after integration over the image domain we end up with the following Bayesian model:

$$
E_{b}\left(\phi, c_{1}, c_{2}\right)=\mathcal{V}\left(\phi, c_{1}, c_{2}\right)+\gamma \int_{\Omega} \frac{(\phi-\tilde{\phi})^{2}}{2 \sigma^{2}} \delta_{\epsilon}(\phi) \mathrm{d} x,
$$

the factor term $\delta_{\epsilon}$ allows us to restrict the shape prior within the region of interest. For a fixed $\phi$, we deduce the values of $c_{1}$ and $c_{2}$ :

$$
c_{1}(\phi)=\frac{\int_{\Omega} u_{0} H_{\epsilon}(\phi) \mathrm{d} x}{\int_{\Omega} H_{\epsilon}(\phi) \mathrm{d} x}, \quad c_{2}(\phi)=\frac{\int_{\Omega} u_{0}\left(1-H_{\epsilon}(\phi)\right) \mathrm{d} x}{\int_{\Omega}\left(1-H_{\epsilon}(\phi)\right) \mathrm{d} x}
$$

As usual, we use an artificial parameter $t$ in the Euler-Lagrange formulation associated to Equation (8) :

$$
\begin{aligned}
& \frac{\partial \phi}{\partial t}=\left(\mu \operatorname{div}\left(\frac{\nabla \phi}{|\nabla \phi|}\right)-\nu-\lambda_{1}\left(u_{0}-c_{1}\right)^{2}+\lambda_{2}\left(u_{0}-c_{2}\right)^{2}\right) \delta_{\epsilon}(\phi)+ \\
& \frac{\gamma}{2 \sigma^{2}}\left(2(\phi-\tilde{\phi}) \delta_{\epsilon}(\phi)+(\phi-\tilde{\phi})^{2} \frac{\partial \delta_{\epsilon}}{\partial \phi}(\phi)\right)=0 \\
& \text { in } \Omega \times \mathbb{R}^{+} ; \phi(x, 0)=\phi_{0}(x) \text { in } \Omega ; \quad \frac{\delta_{\epsilon}(\phi)}{|\nabla \phi|} \frac{\partial \phi}{\partial n}=0 \text { on } \partial \Omega
\end{aligned}
$$

The estimation of the solution of the model (8) can be summarized in the following steps:

- initialize $\phi_{0}=\tilde{\phi}, n=0$;

- compute $c_{1}\left(\phi_{n}\right)$ and $c_{2}\left(\phi_{n}\right)$ by the relations (9);

- compute $\phi_{n+1}$ by solving the PDE (10) with respect to $\phi$;

- update periodically the level set $\phi_{n}$ by a signed distance;

- repeat these three steps until convergence ( $\phi_{n}$ is stationary).

Figure 2 illustrates the segmentation with our approach and shows a comparison with a classical level set method, we will give more detains in the next section. 

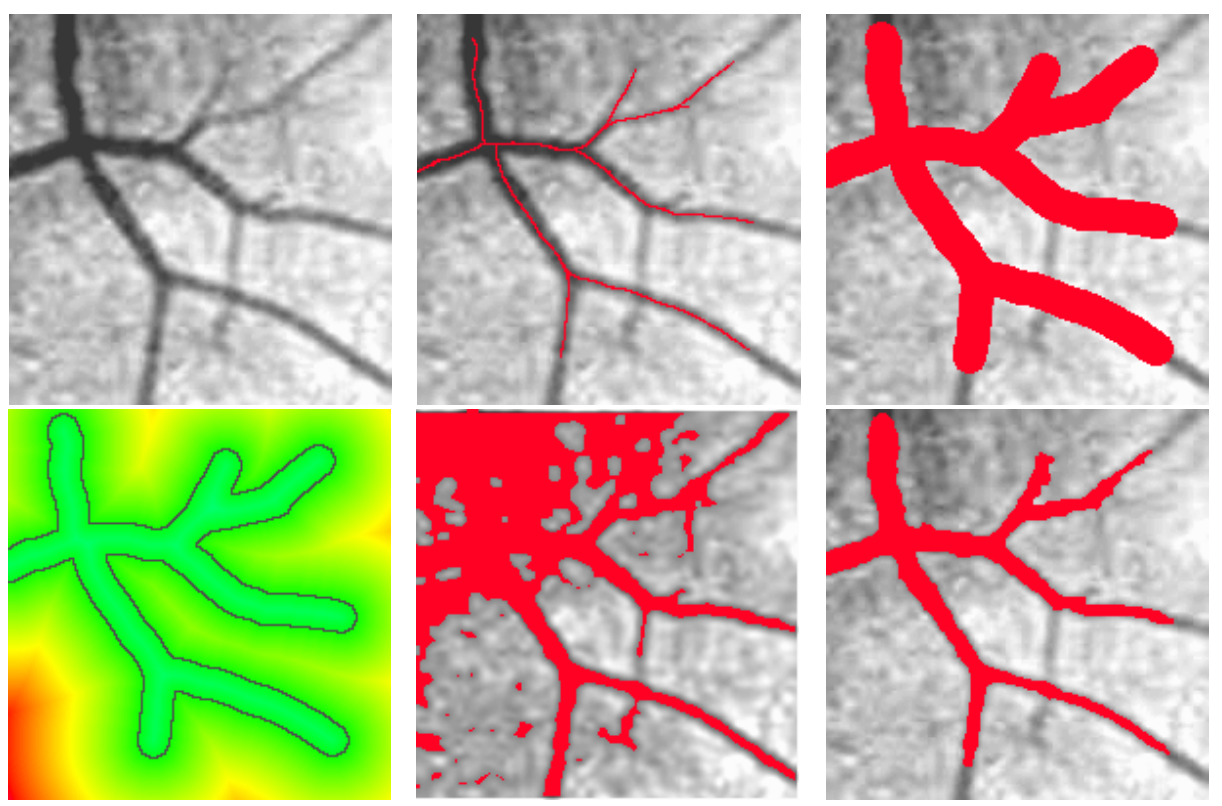

Fig. 2. Segmentation of vessels from a 2D angiogram image. First row: the left panel shows a $2 \mathrm{D}$ angiogram image; the center panel shows in red the voting tree; the right panel shows in red the voting tree after morphological dilatation. Second row: the left panel shows the signed distance computed from the dilated voting tree; the center panel shows in red the segmentation results obtained with a Chan and Vese method without shape prior; the right panel shows the segmentation result obtained with our approach.

\section{Results and Discussion}

We show results obtained with our algorithm on 2D images, see Table 1 and Figure 3, We applied our approach on ten cropped retinal images provided by DRIVE (Digital Retinal Images for Vessel Extraction) 21. The DRIVE data were acquired using a Canon CR5 non-mydriatic 3CCD camera with a 45 degree field of view (FOV). Each image was captured using 8 bits per color plane at 768 by 584 pixels. The FOV of each image is circular with a diameter of approximately 540 pixels. For this database, the images have been cropped around the FOV. The DRIVE data is composed of 40 images for which manual segmentations are also provided. Considering the complexity of the retinal images and the properties of our algorithm, we have cropped ten different images from the $40 \mathrm{im}$ ages availabe and evaluated our method on them. In tables 1 and 1, we compare our approach using the three evaluation measures: Dice, Specificity, and Sensitivity. The maximum value of the Dice index is 1 , which corresponds to a perfect overlap between the manual and automatic segmentations. It shows that the results obtained with our approach are coherent with the manual segmentation. 

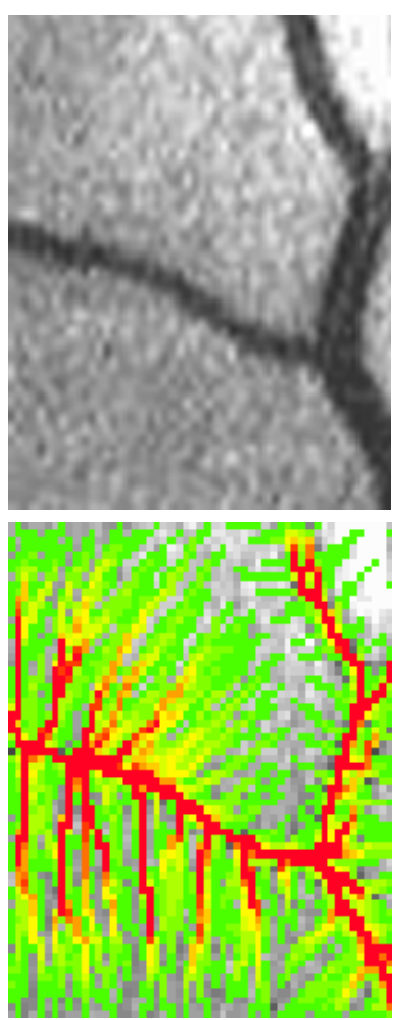
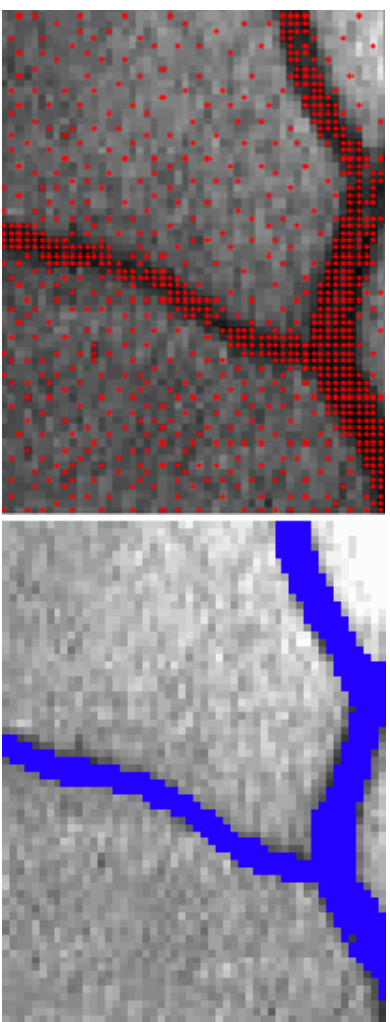
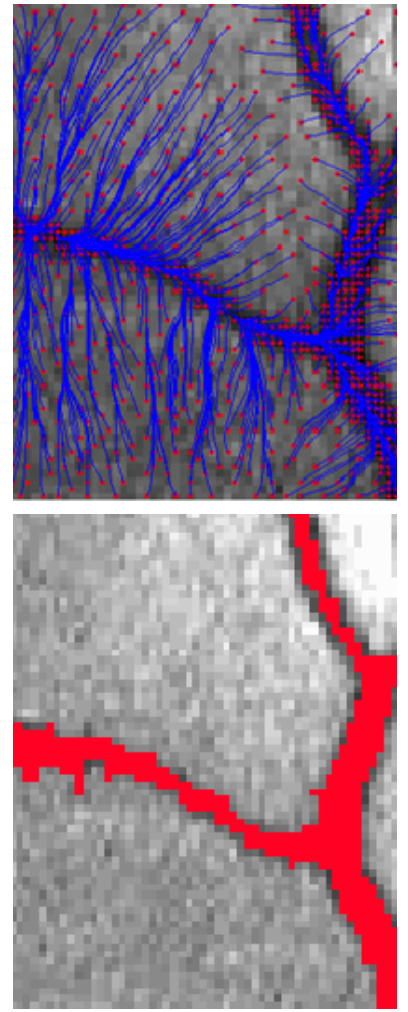

Fig. 3. Segmentation of vessels from one of the ten cropped 2D-retinal images given in table 1. First row: the left panel shows the original image; the center panel shows in red the farthest points detected; the right panel shows in blue the paths extracted from the farthest points to the source point. Second row: the left panel shows the computed geodesic density (green corresponds to a low density and red to a high density); the center panel shows the manual segmentation; the right panel shows the segmentation result obtained with our approach.

For our experiments we have considered the following potential $P(x)=I(x)^{3}$, where $I$ is the grayscale intensity image of the DRIVE images. Figure 3 shows the segmentation result obtained with our approach. The shape prior allows us to constrain the propagation inside the tubular tree. Figure 2 (second row, center column) shows that the propagation without shape constraints $(\gamma=0$ in the Equation (8) ) can leak outside the tree structure.

We have also applied our approach on 3D simulated data of carotid bifurcation lumen created from the simulated data provided by MICCAI challenge [22, by adding Gaussian noise, see figure 4 The results obtained for these simulated data are better than those obtained for the DRIVE data in terms of the following overlap metrics: Dice, sensitivity, and specificity. 

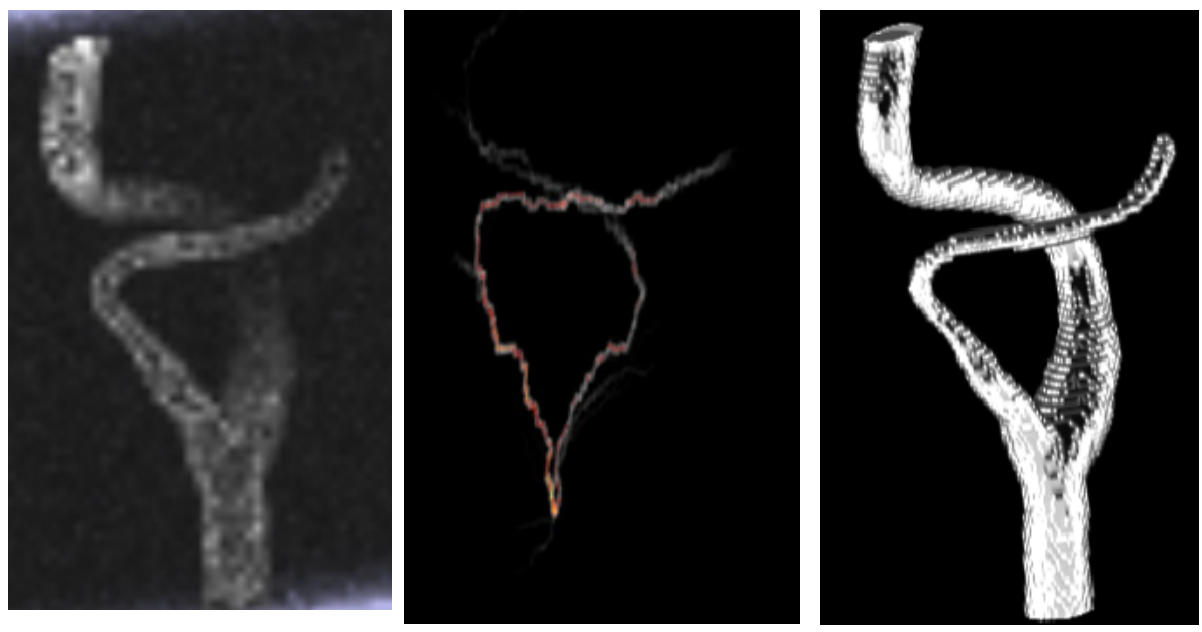

Fig. 4. Lumen segmentation from 3D simulated data (MIP visualization). The left panel shows the original image, the center panel shows the geodesic density, the right panel shows the segmentation result obtained with our approach.

Table 1. Comparison of our segmentations with the manual segmentation, on the ten cropped images from the DRIVE data, in terms of the following statistics: Dice similarity, sensitivity and specificity

\begin{tabular}{|c|c|c|c|c|c|c|c|c|c|c|}
\hline Test data & T1 & T2 & T3 & T4 & T5 & T6 & T7 & T8 & T9 & T10 \\
\hline Dice index & 0.93 & 0.73 & 0.73 & 0.72 & 0.67 & 0.71 & 0.79 & 0.78 & 0.73 & 0.80 \\
\hline Sensitivity & 0.91 & 0.61 & 0.64 & 0.58 & 0.53 & 0.60 & 0.70 & 0.70 & 0.70 & 0.70 \\
\hline Specificity & 0.95 & 0.90 & 0.83 & 0.95 & 0.90 & 0.95 & 0.90 & 0.88 & 0.93 & 0.78 \\
\hline
\end{tabular}

Table 2. Mean and standard deviation values of the statistics: Dice measure, sensitivity, and specificity, for all the data test

\begin{tabular}{|c|c|c|c|}
\hline Statistics & Dice measure & Sensitivity & Specificity \\
\hline Mean & 0.76 & 0.67 & 0.90 \\
\hline Standard deviation & 0.07 & 0.10 & 0.05 \\
\hline
\end{tabular}

\section{Conclusion}

In this paper we have presented a new method for the segmentation of tree structures. This method is adapted to segment automatically tubular tree structure from a single point given by the user, no a priori information about the tree is required. In contrast, the methods previously described in the literature for the segmentation of tree structures are not fully automatic and require a priori information of the tree to be segmented. We have applied our approach to 
segment tubular tree structures from 2D retinal images and compared it with the manual segmentation on ten images. The next step is to validate our approach in $3 \mathrm{D}$ on a large data set.

\section{References}

1. Frangi, A.F., Niessen, W.J., Hoogeveen, R.M., van Walsum, T., Viergever, M.A.: Model-based quantitation of 3D magnetic resonance angiographic images. IEEE Trans. Med. Imaging 18(10), 946-956 (1999)

2. Bouix, S., Siddiqi, K., Tannenbaum, A.: Flux driven automatic centerline extraction. Medical Image Analysis 9(3), 209-221 (2005)

3. Li, H., Yezzi, A.: Vessels as 4D curves: Global minimal 4D paths to extract 3D tubular surfaces and centerlines. IEEE Trans. Med. Imaging 26, 1213-1223 (2007)

4. Li, H., Yezzi, A., Cohen, L.: 3D multi-branch tubular surface and centerline extraction with 4D iterative key points. In: Yang, G.-Z., Hawkes, D., Rueckert, D., Noble, A., Taylor, C. (eds.) MICCAI (1) 2009. LNCS, vol. 5762, pp. 1042-1050. Springer, Heidelberg (2009)

5. Kirbas, C., Quek, F.: A review of vessel extraction techniques and algorithms. ACM Comput. Surv. 36(2), 81-121 (2004)

6. Lesage, D., Angelini, E.D., Bloch, I., Funka-Lea, G.: A review of 3D vessel lumen segmentation techniques: Models, features and extraction schemes. Medical Image Analysis 13(6), 819-845 (2009)

7. Avants, B.B., Williams, J.P.: An adaptive minimal path generation technique for vessel tracking in CTA/CE-MRA volume images. In: Delp, S.L., DiGoia, A.M., Jaramaz, B. (eds.) MICCAI 2000. LNCS, vol. 1935, pp. 707-716. Springer, Heidelberg (2000)

8. Deschamps, T., Cohen, L.D.: Minimal paths in 3D images and application to virtual endoscopy. In: Vernon, D. (ed.) ECCV(2) 2000. LNCS, vol. 1843, pp. 543-557. Springer, Heidelberg (2000)

9. Deschamps, T., Cohen, L.D.: Fast extraction of minimal paths in 3D images and applications to virtual endoscopy. Medical Image Analysis 5(4), 281-299 (2001)

10. Gülsün, M.A., Tek, H.: Robust vessel tree modeling. In: Metaxas, D., Axel, L., Fichtinger, G., Székely, G. (eds.) MICCAI (1) 2008, Part I. LNCS, vol. 5241, pp. 602-611. Springer, Heidelberg (2008)

11. Wink, O., Niessen, W.J., Verdonck, B., Viergever, M.A.: Vessel axis determination using wave front propagation analysis. In: Niessen, W.J., Viergever, M.A. (eds.) MICCAI 2001. LNCS, vol. 2208, pp. 845-853. Springer, Heidelberg (2001)

12. Cohen, L.D., Deschamps, T.: Segmentation of 3D tubular objects with adaptive front propagation and minimal tree extraction for 3D medical imaging. Computer Methods in Biomechanics and Biomedical Engineering 10(4) (2007)

13. Rouchdy, Y., Cohen, L.D.: Image segmentation by geodesic voting. application to the extraction of tree structures from confocal microscope images. In: The 19th International Conference on Pattern Recognition, Tampa, Florida, pp. 1-5 (2008)

14. Rouchdy, Y., Cohen, L.D.: The shading zone problem in geodesic voting and its solutions for the segmentation of tree structures. application to the segmentation of microglia extensions. In: Computer Vision and Pattern Recognition Workshop MMBIA, Miami, Florida, pp. 66-71 (2009)

15. Cohen, L.D., Kimmel, R.: Global minimum for active contour models: A minimal path approach. International Journal of Computer Vision 24(1), 57-78 (1997) 
16. Sethian, J.A.: Level set methods and fast marching methods. Cambridge University Press, Cambridge (1999)

17. Chan, T.F., Vese, L.A.: Active contours without edges. IEEE Trans. Med. Imaging 10(2), 266-277 (2001)

18. Mumford, D., Shah, J.: Optimal approximations by piecewise smooth functions and associated variational problems. Communications on Pure and Applied Mathematics 42(5), 577-685 (1989)

19. Leventon, M.E., Faugeras, O.D., Grimson, W.E.L., Wells III, W.E.: Level set based segmentation with intensity and curvature prior. In: MMBIA, pp. 4-11 (2000)

20. Cremers, D., Rousson, M., Deriche, R.: A review of statistical approaches to level set segmentation: Integrating color, texture, motion and shape. International Journal of Computer Vision 72, 215 (2007)

21. Staal, J.J., Abramoff, M.D., Niemeijer, M., Viergever, M.A., van Ginneken, B.: Ridge based vessel segmentation in color images of the retina. IEEE Trans. Med. Imaging 23(4), 501-509 (2004)

22. Hameeteman, K., Freiman, M., Zuluaga, M.A., Joskowicz, L., Rozie, S., van Gils, M.J., van den Borne, L., Sosna, J., Berman, P., Cohen, N., Douek, P., Snchez, I., Aissat, M., van der Lugt, A., Krestin, G.P., Niessen, W.J., van Walsum, T.: Carotid lumen segmentation and stenosis grading challenge. In: MICCAI 2009 (2009) 